\title{
Third Hankel determinant for reciprocal of bounded turning function has a positive real part of order alpha
}

\author{
Bolineni Venkateswarlu and Nekkanti Rani
}

\begin{abstract}
The objective of this paper is to obtain an upper bound to the third Hankel determinant denoted by $\left|H_{3}(1)\right|$ for certain subclass of univalent functions, using Toeplitz determinants.

Mathematics Subject Classification (2010): 30C45, 30C50.

Keywords: Univalent function, upper bound, function whose reciprocal derivative has a positive real part, Hankel determinant, positive real function, Toeplitz determinants.
\end{abstract}

\section{Introduction}

Let $A$ denote the class of all functions $f(z)$ of the form

$$
f(z)=z+\sum_{n=2}^{\infty} a_{n} z^{n}
$$

in the open unit disc $E=\{z:|z|<1\}$. Let $S$ be the subclass of $A$ consisting of univalent functions. For a univalent function in the class $A$, it is well known that the $n^{t h}$ coefficient is bounded by $n$. The bounds for the coefficients give information about the geometric properties of these functions In particular, the growth and distortion properties of a normalized univalent function are determined by the bound of its second coefficient. The Hankel determinant of $f$ for $q \geq 1$ and $n \geq 1$ was defined by Pommerenke [12] as

$$
H_{q}(n)=\left|\begin{array}{cccc}
a_{n} & a_{n+1} & \cdots & a_{n+q-1} \\
a_{n+1} & a_{n+2} & \cdots & a_{n+q} \\
\vdots & \vdots & \vdots & \vdots \\
a_{n+q-1} & a_{n+q} & \cdots & a_{n+2 q-2}
\end{array}\right| .
$$


This determinant has been considered by many authors in the literature. For example, Noor [10] determined the rate of growth of $H_{q}(n)$ as $n \rightarrow \infty$ for the functions in $S$ with bounded boundary. Ehrenborg [4] studied the Hankel determinant of exponential polynomials. The Hankel transform of an integer sequence and some of its properties were discussed by Layman in [7]. In the recent years several authors have investigated bounds for the Hankel determinant of functions belonging to various subclasses of univalent and multivalent analytic functions. In particular for, $q=2, n=1, a_{1}=1$ and $q=2, n=2, a_{1}=1$, the Hankel determinant simplifies respectively to

$$
H_{2}(1)=\left|\begin{array}{ll}
a_{1} & a_{2} \\
a_{2} & a_{3}
\end{array}\right|=a_{3}-a_{2}^{2} \text {, and } H_{2}(2)=\left|\begin{array}{ll}
a_{2} & a_{3} \\
a_{3} & a_{4}
\end{array}\right|=a_{2} a_{4}-a_{3}^{2} .
$$

For our discussion in this paper, we consider the Hankel determinant in the case of $q=3$ and $n=1$, denoted by $H_{3}(1)$, given by

$$
H_{3}(1)=\left|\begin{array}{lll}
a_{1} & a_{2} & a_{3} \\
a_{2} & a_{3} & a_{4} \\
a_{3} & a_{4} & a_{5}
\end{array}\right| .
$$

For $f \in A, a_{1}=1$, so that, we have

$$
H_{3}(1)=a_{3}\left(a_{2} a_{4}-a_{3}^{2}\right)-a_{4}\left(a_{4}-a_{2} a_{3}\right)+a_{5}\left(a_{3}-a_{2}^{2}\right)
$$

and by applying triangle inequality, we obtain

$$
\left|H_{3}(1)\right| \leq\left|a_{3}\right|\left|a_{2} a_{4}-a_{3}^{2}\right|+\left|a_{4}\right|\left|a_{2} a_{3}-a_{4}\right|+\left|a_{5}\right|\left|a_{3}-a_{2}^{2}\right| .
$$

The sharp upper bound to the second Hankel functional $\left|H_{2}(2)\right|$ for the subclass $R T$ of $S$, consisting of functions whose derivative has a positive real part, studied by Mac Gregor [9] was obtained by Janteng [6]. It was known that if $f \in R T$ then $\left|a_{k}\right| \leq \frac{2}{k}$, for $k \in\{2,3, \ldots$.$\} . Further, the best possible sharp upper bound for the$ functional $\left|a_{2} a_{3}-a_{4}\right|$ and $\left|a_{3}-a_{2}^{2}\right|$ was obtained by Babalola [2] and hence the sharp inequality for $\left|H_{3}(1)\right|$, for the class $R T$. For $f \in R T(\alpha)$, the sharp upper bound to second Hankel [14] and $\left|H_{3}(1)\right|$ were obtained by Vamshee Krishna et al.[15]. The sharp upper bound to $H_{3}(1)$ for the subclass of $\widetilde{R T}$ of $S$ consisting of a function whose reciprocal derivative has a positive real part was obtained by Venkateswarlu $[16]$.

Motivated by the result obtained by Babalola [2], we obtain an upper bound to the functional second Hankel determinant, $\left|a_{2} a_{3}-a_{4}\right|$ and hence $\left|H_{3}(1)\right|$, for the function $f$ given in (1.1), when it belongs to the class $\widetilde{R T}(\alpha)$, defined as follows.

Definition 1.1. A function $f(z) \in A$ is said to be function whose reciprocal derivative has a positive real part of order $\alpha$, (also called reciprocal of bounded turning function of order $\alpha)$, denoted by $f \in \widetilde{R T}(\alpha)(0 \leq \alpha<1)$, if and only if

$$
\operatorname{Re}\left(\frac{1}{f^{\prime}(z)}\right)>\alpha, \forall z \in E \text {. }
$$

Observe that for $\alpha=0$, we obtain $\widetilde{R T}(0)=\widetilde{R T}$. Some preliminary lemmas required for proving our results are as follows: 


\section{Preliminary results}

Let $\mathscr{P}$ denote the class of functions consisting of $p$, such that

$$
p(z)=1+c_{1} z+c_{2} z^{2}+c_{3} z^{3}+\ldots=\left[1+\sum_{n=1}^{\infty} c_{n} z^{n}\right],
$$

which are regular in the open unit disc $E$ and satisfy $\operatorname{Re}\{p(z)\}>0$ for any $z \in E$. Here $p(z)$ is called the Caratheòdory function [3].

Lemma 2.1. [11, 13] If $p \in \mathscr{P}$, then $\left|c_{k}\right| \leq 2$, for each $k \geq 1$ and the inequality is sharp for the function $\frac{1+z}{1-z}$.

Lemma 2.2. [5] The power series for $p(z)=1+\sum_{n=1}^{\infty} c_{n} z^{n}$ given in (2.1) converges in the open unit disc $E$ to a function in $\mathscr{P}$ if and only if the Toeplitz determinants

$$
D_{n}=\left|\begin{array}{ccccc}
2 & c_{1} & c_{2} & \cdots & c_{n} \\
c_{-1} & 2 & c_{1} & \cdots & c_{n-1} \\
c_{-2} & c_{-1} & 2 & \cdots & c_{n-2} \\
\vdots & \vdots & \vdots & \vdots & \vdots \\
c_{-n} & c_{-n+1} & c_{-n+2} & \cdots & 2
\end{array}\right|, n=1,2,3, \cdots
$$

and $c_{-k}=\bar{c}_{k}$, are all non-negative. They are strictly positive except for

$$
p(z)=\sum_{k=1}^{m} \rho_{k} p_{0}\left(e^{i t_{k}} z\right)
$$

$\rho_{k}>0, t_{k}$ real and $t_{k} \neq t_{j}$, for $k \neq j$, where $p_{0}(z)=\frac{1+z}{1-z}$; in this case $D_{n}>0$ for $n<(m-1)$ and $D_{n} \doteq 0$ for $n \geq m$.

This necessary and sufficient condition found in [5] is due to Caratheòdory and Toeplitz. We may assume without restriction that $c_{1}>0$. On using Lemma 2.2, for $n=2$, we have

$$
D_{2}=\left|\begin{array}{ccc}
2 & c_{1} & c_{2} \\
\bar{c}_{1} & 2 & c_{1} \\
\bar{c}_{2} & \bar{c}_{1} & 2
\end{array}\right|=\left[8+2 \operatorname{Re}\left\{c_{1}^{2} c_{2}\right\}-2\left|c_{2}\right|^{2}-4\left|c_{1}\right|^{2}\right] \geq 0
$$

which is equivalent to

$$
2 c_{2}=c_{1}^{2}+x\left(4-c_{1}^{2}\right), \text { for some } x,|x| \leq 1 .
$$

For $n=3$,

and is equivalent to

$$
D_{3}=\left|\begin{array}{cccc}
2 & c_{1} & c_{2} & c_{3} \\
\bar{c}_{1} & 2 & c_{1} & c_{2} \\
\bar{c}_{2} & \bar{c}_{1} & 2 & c_{1} \\
\bar{c}_{3} & \bar{c}_{2} & \bar{c}_{1} & 2
\end{array}\right| \geq 0
$$

$$
\left|\left(4 c_{3}-4 c_{1} c_{2}+c_{1}^{3}\right)\left(4-c_{1}^{2}\right)+c_{1}\left(2 c_{2}-c_{1}^{2}\right)^{2}\right| \leq 2\left(4-c_{1}^{2}\right)^{2}-2\left|\left(2 c_{2}-c_{1}^{2}\right)\right|^{2} .
$$


From the relations (2.2) and (2.3), after simplifying, we get

$$
4 c_{3}=c_{1}^{3}+2 c_{1}\left(4-c_{1}^{2}\right) x-c_{1}\left(4-c_{1}^{2}\right) x^{2}+2\left(4-c_{1}^{2}\right)\left(1-|x|^{2}\right) z,
$$

for some $z$, with $|z| \leq 1$.

To obtain our result, we refer to the classical method initiated by Libera and Zlotkiewicz [8] and used by several authors in the literature.

\section{Main result}

Theorem 3.1. If $f(z) \in \widetilde{R T}(\alpha)\left(0 \leq \alpha \leq \frac{1}{\sqrt{2}}\right)$ then

$$
\left|a_{2} a_{4}-a_{3}^{2}\right| \leq\left[\frac{2}{3(\alpha-1)}\right]^{2}
$$

and the inequality is sharp.

Proof. For

$$
f(z)=z+\sum_{n=2}^{\infty} a_{n} z^{n} \in \widetilde{R T}(\alpha)
$$

there exists an analytic function $p \in \mathscr{P}$ in the open unit $\operatorname{disc} E$ with $p(0)=1$ and $\operatorname{Re}\{p(z)\}>0$ such that

$$
\frac{1-\alpha f^{\prime}(z)}{(1-\alpha) f^{\prime}(z)}=p(z) \Leftrightarrow 1-\alpha f^{\prime}(z)=(1-\alpha) f^{\prime}(z) p(z) .
$$

Replacing $f^{\prime}(z)$ and $p(z)$ with their equivalent series expressions in (3.1), we have

$$
1-\alpha\left(1+\sum_{n=2}^{\infty} n a_{n} z^{n-1}\right)=(1-\alpha)\left(1+\sum_{n=2}^{\infty} n a_{n} z^{n}\right)\left(1+\sum_{n=1}^{\infty} c_{n} z^{n}\right) .
$$

Upon simplification, we obtain

$$
\begin{aligned}
& (1-\alpha)-2 \alpha a_{2} z-3 \alpha a_{3} z^{2}-4 \alpha a_{4} z^{3}-5 a_{5} z^{4}-\cdots=(1-\alpha) \\
& +z(1-\alpha)\left[2 a_{2}+c_{1}\right]+z^{2}(1-\alpha)\left[c_{2}+2 a_{2} c_{1}+3 a_{3}\right]+z^{3}(1-\alpha) \\
& {\left[c_{3}+2 a_{2} c_{2}+3 a_{3} c_{1}+4 a_{4}\right]+z^{4}(1-\alpha)\left[c_{4}+2 a_{2} c_{3}+3 a_{3} c_{2}+4 a_{4} c_{1}+5 a_{5}\right]+\cdots .}
\end{aligned}
$$

Equating the coefficients of like powers of $z, z^{2}, z^{3}$ and $z^{4}$ respectively on both sides of (3.2), after simplifying, we get

$$
\begin{aligned}
& a_{2}=-\frac{1-\alpha}{2} c_{1} ; a_{3}=-\frac{1-\alpha}{3}\left[c_{2}-(1-\alpha) c_{1}^{2}\right] \\
& a_{4}=-\frac{1-\alpha}{4}\left[c_{3}-2(1-\alpha) c_{1} c_{2}+(1-\alpha)^{2} c_{1}^{3}\right] \\
& a_{5}=-\frac{1-\alpha}{5}\left[c_{4}-2(1-\alpha) c_{1} c_{3}+3(1-\alpha)^{2} c_{1}^{2} c_{2}-(1-\alpha) c_{2}^{2}-(1-\alpha)^{3} c_{1}^{4}\right] .
\end{aligned}
$$


Substituting the values of $a_{2}, a_{3}$ and $a_{4}$ from (3.3) in the functional $\left|a_{2} a_{4}-a_{3}^{2}\right|$ for the function $f \in \widetilde{R T}(\alpha)$, upon simplification, we obtain

$$
\left|a_{2} a_{4}-a_{3}^{2}\right|=\frac{(1-\alpha)^{2}}{72}\left|9 c_{1} c_{3}-2(1-\alpha) c_{1}^{2} c_{2}-8 c_{2}^{2}+(1-\alpha)^{2} c_{1}^{4}\right|
$$

which is equivalent to

$$
\begin{aligned}
& \left|a_{2} a_{4}-a_{3}^{2}\right|=\frac{(1-\alpha)^{2}}{72}\left|d_{1} c_{1} c_{3}+d_{2} c_{1}^{2} c_{2}+d_{3} c_{2}^{2}+d_{4} c_{1}^{4}\right| \\
& \text { where } d_{1}=9 ; d_{2}=-2(1-\alpha) ; d_{3}=-8 ; d_{4}=(1-\alpha)^{2}
\end{aligned}
$$

Substituting the values of $c_{2}$ and $c_{3}$ given in (2.2) and (2.4) respectively from Lemma 2.2 on the right-hand side of (3.4), we have

$$
\begin{aligned}
\left|d_{1} c_{1} c_{3}+d_{2} c_{1}^{2} c_{2}+d_{3} c_{2}^{2}+d_{4} c_{1}^{4}\right| & =\mid \frac{d_{1} c_{1}}{4}\left\{c_{1}^{3}+2 c_{1}\left(4-c_{1}^{2}\right) x-c_{1}\left(4-c_{1}^{2}\right) x^{2}\right. \\
& \left.+2\left(4-c_{1}^{2}\right)\left(1-|x|^{2}\right) z\right\}+\frac{d_{2} c_{1}^{2}}{2}\left\{c_{1}^{2}+x\left(4-c_{1}^{2}\right)\right\} \\
& +\frac{d_{3}}{4}\left\{c_{1}^{2}+x\left(4-c_{1}^{2}\right)\right\}^{2}+d_{4} c_{1}^{4} \mid
\end{aligned}
$$

Using triangle inequality and the fact that $|z|<1$, we get

$$
\begin{aligned}
4\left|d_{1} c_{1} c_{3}+d_{2} c_{1}^{2} c_{2}+d_{3} c_{2}^{2}+d_{4} c_{1}^{4}\right| & \leq \mid\left(d_{1}+2 d_{2}+d_{3}+4 d_{4}\right) c_{1}^{4}+2 d_{1} c_{1}\left(4-c_{1}^{2}\right) \\
& +2\left(d_{1}+d_{2}+d_{3}\right) c_{1}^{2}\left(4-c_{1}^{2}\right)|x| \\
& -\left\{\left(d_{1}+d_{3}\right) c_{1}^{2}+2 d_{1} c_{1}-4 d_{3}\right\}\left(4-c_{1}^{2}\right)|x|^{2} \mid .
\end{aligned}
$$

From (3.5), we can now write

$$
\begin{array}{r}
d_{1}+2 d_{2}+d_{3}+4 d_{4}=4 \alpha^{2}-4 \alpha+1 ; 2\left(d_{1}+d_{2}+d_{3}\right)=-2(1-2 \alpha) \\
\left(d_{1}+d_{3}\right) c_{1}^{2}+2 d_{1} c_{1}-4 d_{3}=c_{1}^{2}+18 c_{1}+32=\left(c_{1}+16\right)\left(c_{1}+2\right) .
\end{array}
$$

Since $c_{1} \in[0,2]$, using the result $\left(c_{1}+a\right)\left(c_{1}+b\right) \geq\left(c_{1}-a\right)\left(c_{1}-b\right)$, where $a, b \geq 0$ in (3.9), we can have

$$
-\left\{\left(d_{1}+d_{3}\right) c_{1}^{2}+2 d_{1} c_{1}-4 d_{3}\right\} \leq-\left(c_{1}^{2}-18 c_{1}+32\right) .
$$

Substituting the calculated values from (3.8) and (3.10) on the right-hand side of (3.7), we have

$$
\begin{aligned}
4 \mid d_{1} c_{1} c_{3}+d_{2} c_{1}^{2} c_{2} & +d_{3} c_{2}^{2}+d_{4} c_{1}^{4}|\leq|\left(4 \alpha^{2}-4 \alpha+1\right) c_{1}^{4}+18 c_{1}\left(4-c_{1}^{2}\right) \\
& -2(1-2 \alpha) c_{1}^{2}\left(4-c_{1}^{2}\right)|x|-\left(c_{1}^{2}-18 c_{1}+32\right)\left(4-c_{1}^{2}\right)|x|^{2} \mid
\end{aligned}
$$


Choosing $c_{1}=c \in[0,2]$, applying triangle inequality and replacing $|x|$ by $\mu$ on the right-hand side of the above inequality, we get

$$
\begin{aligned}
4 \mid d_{1} c_{1} c_{3}+d_{2} c_{1}^{2} c_{2} & +d_{3} c_{2}^{2}+d_{4} c_{1}^{4} \mid \leq\left[\left(4 \alpha^{2}-4 \alpha+1\right) c^{4}+18 c\left(4-c^{2}\right)\right. \\
& \left.+2(1-2 \alpha) c^{2}\left(4-c^{2}\right) \mu+\left(c^{2}-18 c+32\right)\left(4-c^{2}\right) \mu^{2}\right] \\
& =F(c, \mu), 0 \leq \mu=|x| \leq 1 \text { and } 0 \leq c \leq 2 .
\end{aligned}
$$

We next maximize the function $F(c, \mu)$ on the closed region $[0,2] \times[0,1]$. Differentiating $F(c, \mu)$ given in (3.12) partially with respect to $\mu$, we obtain

$$
\frac{\partial F}{\partial \mu}=2\left[(1-2 \alpha) c^{2}+\left(c^{2}-18 c+32\right) \mu\right]\left(4-c^{2}\right) .
$$

For $0<\mu<1$ and for fixed $c$ with $0<c<2$, from (3.13), we observe that $\frac{\partial F}{\partial \mu}>0$. Therefore, $F(c, \mu)$ becomes an increasing function of $\mu$ and hence it cannot have a maximum value at any point in the interior of the closed region $[0,2] \times[0,1]$. Moreover, for a fixed $c \in[0,2]$, we have

$$
\max _{0 \leq \mu \leq 1} F(c, \mu)=F(c, 1)=G(c) .
$$

Therefore, replacing $\mu$ by 1 in $F(c, \mu)$, upon simplification, we obtain

$$
\begin{aligned}
& \left.G(c)=2\left[-c^{4}\left(1-2 \alpha^{2}\right)-2 c^{2}(4 \alpha+5)+64\right)\right] . \\
& G^{\prime}(c)=-8 c\left[c^{2}\left(1-2 \alpha^{2}\right)+(4 \alpha+5)\right] .
\end{aligned}
$$

From (3.15), we observe that $G^{\prime}(c) \leq 0$, for every $c \in[0,2]$. Therefore, $G(c)$ is a decreasing function of $c$ in the interval $[0,2]$, whose maximum value occurs at $c=0$ only. From (3.14), the maximum value of $G(c)$ at $c=0$ is given by

$$
G_{\max }=G(0)=128 .
$$

Simplifying the expressions (3.12) and (3.16), we get

$$
\left|d_{1} c_{1} c_{3}+d_{2} c_{1}^{2} c_{2}+d_{3} c_{2}^{2}+d_{4} c_{1}^{4}\right| \leq 32 .
$$

From the relations (3.4) and (3.17), upon simplification, we obtain

$$
\left|a_{2} a_{4}-a_{3}^{2}\right| \leq\left[\frac{2}{3}(1-\alpha)\right]^{2}
$$

By setting $c_{1}=c=0$ and selecting $x=1$ in the expressions (2.2) and (2.4), we find that $c_{2}=2$ and $c_{3}=0$ respectively. Substituting these values in (3.17) together with the values in (3.4), we observe that equality is attained, which shows that our result is sharp. The extremal function in this case is given by

$$
\frac{1-\alpha f^{\prime}(z)}{(1-\alpha) f^{\prime}(z)}=1+2 z^{2}+2 z^{4}+\cdots=\frac{1+z^{2}}{1-z^{2}} .
$$

This completes the proof of our Theorem. 
Remark 3.2. It is observed that the sharp upper bound to the second Hankel determinant of a function whose derivative has a positive real part of order $\alpha$, obtained by Vamshee Krishna et al. [14] and a function whose reciprocal derivative has a positive real part of order $\alpha$ is the same. Further, for the choice of $\alpha=0$, we get $\widetilde{R T}(0)=\widetilde{R T}$, for which from (3.18), we obtain $\left|a_{2} a_{4}-a_{3}^{2}\right| \leq \frac{4}{9}$. This inequality is sharp and this result coincides with that of Janteng et al. [6] and Venkateswarlu et al. [16]. From this we conclude that the sharp upper bound to the second Hankel determinant of a function whose derivative has a positive real part of order $\alpha$ and a function whose reciprocal derivative has a positive real part of order $\alpha$ is the same.

Theorem 3.3. If $f(z) \in \widetilde{R T}(\alpha)\left(0 \leq \alpha \leq \frac{5}{8}\right)$ then $\left|a_{2} a_{3}-a_{4}\right| \leq \frac{1}{6}\left[\frac{5-8 \alpha}{3}\right]^{\frac{3}{2}}$.

Proof. Substituting the values of $a_{2}, a_{3}$ and $a_{4}$ from (3.3) in the determinant $\left|a_{2} a_{3}-a_{4}\right|$ for the function $f \in \widetilde{R T}(\alpha)$, after simplifying, we get

$$
\left|a_{2} a_{3}-a_{4}\right|=\frac{(1-\alpha)}{12}\left|3 c_{3}-4(1-\alpha) c_{1} c_{2}+(1-\alpha)^{2} c_{1}^{3}\right| .
$$

Substituting the values of $c_{2}$ and $c_{3}$ from (2.2) and (2.4) respectively from Lemma 2.2 on the right-hand side of (3.19), and using the fact that $|z|<1$, we have

$$
\begin{aligned}
4 \mid 3 c_{3}-4(1-\alpha) c_{1} c_{2} & +(1-\alpha)^{2} c_{1}^{3}|\leq|-c_{1}^{3}\left(1-4 \alpha^{2}\right)+6\left(4-c_{1}^{2}\right) \\
& -2 c_{1}\left(4-c_{1}^{2}\right)|x|(1-4 \alpha)-3\left(4-c_{1}^{2}\right)|x|^{2}\left(c_{1}+2\right) \mid .
\end{aligned}
$$

Since $c_{1}=c \in[0,2]$, using the result $\left(c_{1}+a\right) \geq\left(c_{1}-a\right)$, where $a \geq 0$, applying triangle inequality and replacing $|x|$ by $\mu$ on the right-hand side of the above inequality, we have

$$
\begin{aligned}
4\left|3 c_{3}-4 c_{1} c_{2}+c_{1}^{3}\right| & \leq \mid c^{3}\left(1-4 \alpha^{2}\right)+6\left(4-c^{2}\right) \\
& +2(1-\alpha) c\left(4-c^{2}\right) \mu+3(c-2)\left(4-c^{2}\right) \mu^{2} \mid \\
& =F(c, \mu), 0 \leq \mu=|x| \leq 1 \text { and } 0 \leq c \leq 2
\end{aligned}
$$

Next, we maximize the function $F(c, \mu)$ on the closed square $[0,2] \times[0,1]$.

Differentiating $F(c, \mu)$ given in(3.20) partially with respect to $\mu$, we get

$$
\frac{\partial F}{\partial \mu}=2\left(4-c^{2}\right)[(1-4 \alpha) c+3(c-2) \mu]>0 .
$$

As described in Theorem 3.1, further we obtain

$$
\begin{aligned}
& G(c)=-4 c^{3}(1-\alpha)^{2}+4(5-8 \alpha) c . \\
& G^{\prime}(c)=-12 c^{2}(1-\alpha)^{2}+4(5-8 \alpha) c . \\
& G^{\prime \prime}(c)=-24 c(1-\alpha)^{2} .
\end{aligned}
$$

For optimum value of $G(c)$, consider $G^{\prime}(c)=0$, From (3.23), we get

$$
c^{2}=\frac{5-8 \alpha}{3(1-\alpha)^{2}} \text {, for } 0 \leq \alpha<\frac{5}{8} \text {. }
$$


Using the obtained value of $c=\sqrt{\frac{5-8 \alpha}{3(1-\alpha)^{2}}} \in[0,2]$ in (3.24). In which simplifies to give

$$
G^{\prime \prime}(c)=-24 \sqrt{\frac{5-8 \alpha}{3}(1-\alpha)}<0, \text { for } 0 \leq \alpha<\frac{5}{8} .
$$

Therefore, by the second derivative test, $G(c)$ has maximum value at $c=\sqrt{\frac{5-8 \alpha}{3(1-\alpha)^{2}}}$. Substituting the value of $c$ in the expression (3.22), upon simplification, we obtain the maximum value of $G(c)$ at $c$, as

$$
G_{\max }=\frac{8}{1-\alpha}\left[\frac{5-8 \alpha}{3}\right]^{\frac{3}{2}} .
$$

From the expressions (3.20) and (3.25), after simplifying, we get

$$
\left|3 c_{3}-4(1-\alpha) c_{1} c_{2}+(1-\alpha)^{2} c_{1}^{3}\right| \leq \frac{2}{1-\alpha}\left[\frac{5-8 \alpha}{3}\right]^{\frac{3}{2}} .
$$

Simplifying the relations (3.19) and (3.26), upon simplification, we obtain

$$
\left|a_{2} a_{3}-a_{4}\right| \leq \frac{1}{6}\left[\frac{5-8 \alpha}{3}\right]^{\frac{3}{2}}
$$

This completes the proof of our Theorem.

Remark 3.4. For the choice of $\alpha=0$, from (3.27), we obtain $\left|a_{2} a_{3}-a_{4}\right| \leq \frac{1}{6}\left(\frac{5}{3}\right)^{\frac{3}{2}}$. This inequality is sharp and this result coincides with that of obtained by Babalola [2] and Venkateswarlu et al. [16]. From this we conclude that for $\alpha=0$, the sharp upper bound to the $\left|a_{2} a_{3}-a_{4}\right|$ of a function whose derivative has a positive real part of order alpha and a function whose reciprocal derivative has a positive real part or order alpha is the same.

The following theorem is a straight forward verification on applying the same procedure as described in Theorems 3.1 and 3.3 and the result is sharp for the values $c_{1}=0, c_{2}=2$ and $x=1$.

Theorem 3.5. If $f \in \widetilde{R T}(\alpha)(0 \leq \alpha<1)$ then $\left|a_{3}-a_{2}^{2}\right| \leq \frac{2}{3}[1-\alpha]$.

Using the fact that $\left|c_{n}\right| \leq 2, n \in N=\{1,2,3, \cdots\}$, with the help of $c_{2}$ and $c_{3}$ values given in (2.2) and (2.4) respectively together with the values in (3.3), we obtain $\left|a_{k}\right| \leq \frac{2}{k}(1-\alpha)(1-2 \alpha)^{k-2}$, for $k \in\{2,3,4,5, \cdots\}$.

Substituting the results of Theorems 3.1, 3.3, 3.5 and $\left|a_{k}\right| \leq$ $\frac{2}{k}(1-\alpha)(1-2 \alpha)^{k-2}$, for $k \in\{2,3,4,5, \cdots\}$, for the function $f \in \widetilde{R T}(\alpha)$ in the inequality (1.3), upon simplification, we obtain the following corollary.

Corollary 3.6. If $f(z) \in \widetilde{R T}(\alpha)\left(0 \leq \alpha \leq \frac{1}{\sqrt{2}}\right)$ then

$$
\left|H_{3}(1)\right| \leq \frac{(1-\alpha)(1-2 \alpha)}{3}\left[\frac{4(1-\alpha)\left(36 \alpha^{2}-46 \alpha+19\right)}{45}+\frac{(1-2 \alpha)}{4}\left(\frac{5-8 \alpha}{3}\right)^{\frac{3}{2}}\right] \text {. }
$$


Remark 3.7. We choose $\alpha=0$, from the expressions (3.28), we obtain $\left|H_{3}(1)\right| \leq$ 0.742. These inequalities are sharp and coincide with the results of Babalola [2] and Venkateswarlu et al. [16]. From this we conclude that for $\alpha=0$, the sharp upper bound to the third Hankel determinant of a function whose derivative has a positive real part or order alpha and a function whose reciprocal derivative has a positive real part of order alpha is the same.

\section{References}

[1] Ali, R.M., Coefficients of the inverse of strongly starlike functions, Bull. Malays. Math. Sci. Soc., (second series), 26(2003), no. 1, 63-71.

[2] Babalola, K.O., On $\mathrm{H}_{3}(1)$ Hankel determinant for some classes of univalent functions, Inequ. Theory and Appl., 6(2010), 1-7.

[3] Duren, P.L., Univalent Functions, vol. 259, Grundlehren der Mathematischen Wissenschaften, Springer, New York, USA, 1983.

[4] Ehrenborg, R., The Hankel determinant of exponential polynomials, Amer. Math. Monthly, 107(2000), no. 6, 557-560.

[5] Grenander, U., Szegö, G., Toeplitz Forms and Their Applications, Second Edition, Chelsea Publishing Co., New York, 1984.

[6] Janteng, A., Halim, S.A., Darus, M., Coefficient inequality for a function whose derivative has a positive real part, J. Inequal. Pure Appl. Math., 7(2006), no. 2, 1-5.

[7] Layman, J.W., The Hankel transform and some of its properties, J. Integer Seq., 4(2001), no. 1, 1-11.

[8] Libera, R.J., Zlotkiewicz, E.J., Coefficient bounds for the inverse of a function with derivative in $\mathscr{P}$, Proc. Amer. Math. Soc., 87(1983), no. 2, 251-257.

[9] Mac Gregor, T.H., Functions whose derivative have a positive real part, Trans. Amer. Math. Soc., 104(1962), no. 3, 532 - 537.

[10] Noor, K.I., Hankel determinant problem for the class of functions with bounded boundary rotation, Rev. Roumaine Math. Pures Appl., 28(1983), no. 8, 731-739.

[11] Pommerenke, Ch., Univalent Functions, Vandenhoeck and Ruprecht, Göttingen, 1975.

[12] Pommerenke, Ch., On the coefficients and Hankel determinants of univalent functions, J. Lond. Math. Soc., 41(1966), 111-122.

[13] Simon, B., Orthogonal polynomials on the unit circle, Part 1, Classical theory, Amer. Math. Soc. Colloq. Publ., 54, Part 1, 2005.

[14] Vamshee Krishna, D., RamReddy, T., Coefficient inequality for a function whose derivative has a positive real part of order $\alpha$, Math. Bohem., 140(2015), 43-52.

[15] Vamshee Krishna, D., Venkateswarlu, B., RamReddy, T., Third Hankel determinant for bounded turning functions of order alpha, J. Nigerian Math. Soc., 34(2015), 121-127.

[16] Venkateswarlu, B., Vamshee Krishna, D., Rani, N., RamReddy, T., Third Hankel determinant for reciprocal of bounded turning functions (Communicated).

Bolineni Venkateswarlu

Department of Mathematics, GIT, GITAM University

Visakhapatnam, 530045, A.P., India

e-mail: bvlmaths@gmail.com 
Nekkanti Rani

Department of Sciences and Humanities

Praveenya Institute of Marine Engineering and Maritime studies

Modavalasa, 534 002, Visakhapatnam, A.P., India

e-mail: raninekkanti1111@gmail.com 